
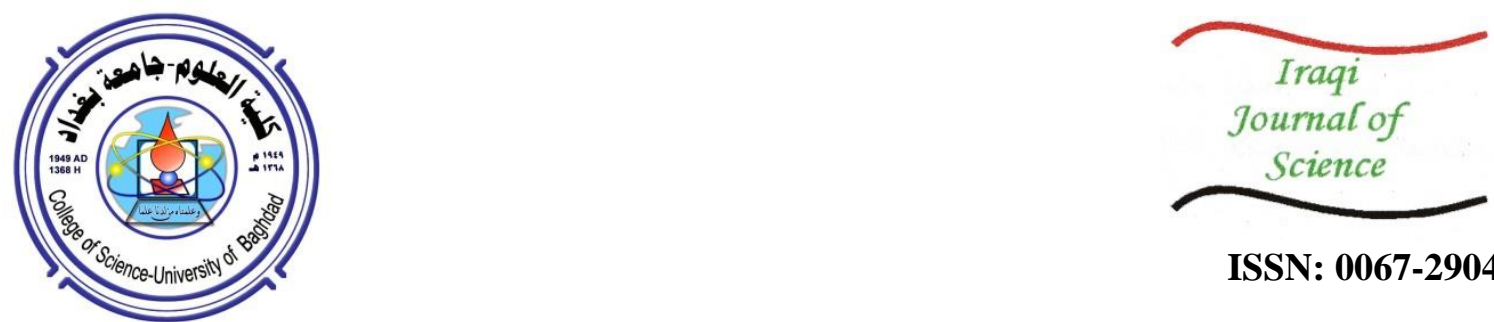

ISSN: 0067-2904

\title{
Antibacterial Susceptibility of Clinical Isolates of Klebsiella pneumoniae in Nigeria to Carbapenems
}

\author{
Oladipo E.K ${ }^{1}$, Awoyelu E.H ${ }^{2}$, Adeosun I.J *1 , Ayandele A.A ${ }^{2}$. \\ ${ }^{1}$ Department of Microbiology, Laboratory of Molecular Biology, Bioinformatics and Immunology, Adeleke \\ University, P.M.B 250, Ede, Osun State, Nigeria. \\ ${ }^{2}$ Department of Pure and Applied Biology (Microbiology/Virology Unit), Ladoke Akintola University, P.M.B \\ 4000, Ogbomoso, Nigeria
}

Received: 5/4/2020

Accepted: 8/5/2020

\begin{abstract}
The emergent bacterial resistance to antibiotics, most especially Carbapenems, has become a common phenomenon. The aim of the recent study was the observation and evaluation of the antibacterial susceptibility of Klebsiella pneumoniae in clinical specimens to different Carbapenems. One hundred isolates of $K$. pneumoniae isolated from different clinical sites, such as leg, caesarean section (CS), head, buttock, breast, thigh, and arm were tested. Using disc diffusion method, the isolates were tested for susceptibility to different antibiotics including Tobramycin, Ciprofloxacin, Aztreonam, Colistin sulphate, Ceftriaxone, Cefepime, Cefoxitin, Ceftazidime, Ertapenem, Meropenem, and Imipenem. The results were interpreted according to the Clinical and Laboratory Standard Institute disk diffusion standard. All K. pneumoniae isolates were highly susceptible to all classes of Carbapenems: Imipenem (99\%), Meropenem (96\%) and Ertapenem (91\%). However, they were highly resistant to Ciprofloxacin (97\%), Ceftriaxone (91\%) and Tobramycin (73\%). Despite the recent emergence of multi-drug resistant bacteria to Carbapenems, this study showed that Carbapenems could still be used in treating different infections caused by multi-drug resistant $K$. pneumoniae.
\end{abstract}

Keywords: Antibiotic resistance, Aminoglycosides, Carbapenems, Clinical isolates, Klebsiella pneumoniae

\section{Introduction}

K. pneumoniae, a Gram-negative bacterium, has been recognized to be clinically relevant in that it has the ability to acquire multidrug resistance, thus limiting therapeutic options for treating infections, thereby posing a serious threat globally, particularly in Nigeria [1].

The usual antibiotic treatments for $K$. pneumoniae infections include $\beta$-lactams such as cephalosporins and carbapenems, aminoglycosides such as gentamycin, and quinolones [2]. These treatments, however, are ineffective against certain strains of $K$. pneumoniae that contain effective resistance mechanisms. K. pneumoniae have various mechanisms of resistance, including those shown by Gram-negative bacteria against various antibiotics, such as Aminoglycosides, Monobactams and Cephems. These mechanisms involve loss of porins, that results in reducing the movement of drug through the cell membrane, production of enzymes [3], and the presence of $\beta$-lactamases in the periplasmic space, which degrades the $\beta$-lactam. They also involve increased expression of the transmembrane efflux pump, which expels the drug from the bacterium, target site mutations, which prevents the antibiotic from binding to its site of action, and ribosomal mutations or modifications, which prevent the antibiotic from binding and inhibiting protein synthesis. Other examples of such mechanisms include biofilm formation, which protects the $K$. pneumoniae from antibiotic treatments

*Email: adeosunidowu6@gmail.com 
[4], metabolic bypass mechanisms, which use an alternative resistant enzyme to bypass the inhibitory effect of the antibiotic, and a mutation in the lipopolysaccharide, which renders the polymyxin class of antibiotics unable to bind this target [5].

$K$. pneumoniae also produces various enzymes that target and deactivate specific parts of drugs [6, 7]. The enzymes produced usually target beta lactam type drugs, but some target other drug classes. These enzymes include extended spectrum beta lactamases, metallo-beta-lactamases, oxacillinases, $K$. pneumoniae carbapenemases, and various others [7].

Carbapenems, on the other hand, are a class of $\beta$-lactam, broad spectrum antibiotics which act by inhibiting the cell wall synthesis. They possess the ability to be stable to hydrolysis by most beta lactamases, hence their use as a "last resort" for treatment of infections. They are known to be particularly effective against Escherichia coli and K. pneumoniae, despite great public concern about the increasing incidence of Carbapenems-resistant strains [8].

In Nigeria, there has been increasing reports on Carbapenem-resistant $K$. pneumoniae together with other antibiotics. However, the current study was carried out to evaluate the effectiveness of Carbapenems on clinical isolates of $K$. pneumoniae.

\section{Methods}

A total of one hundred isolates of K. pneumoniae isolated from wounds of different clinical sites such as leg, caesarean section (CS), head, buttock, breast, thigh and arm of humans were collected from the Department of Medical Microbiology and Parasitology of The University College Hospital (UCH), Ibadan, Oyo State, Nigeria. The isolates were inoculated into nutrient agar slants, labelled properly, and incubated at $37^{\circ} \mathrm{C}$ for 24 hours.

\section{Conventional identification of $K$. pneumoniae isolates}

Clinical isolates of $K$. pneumoniae were identified using the conventional method described by Cheesbrough [9]. This involved carrying out gram staining test, catalase test, urease test, oxidase test, and coagulase test on the pre-identified Klebsiella pneumoniae colonies [9].

\section{Antimicrobial susceptibility testing}

Susceptibility testing was carried out on K. pneumoniae using Kirby-Bauer disc diffusion method. The methods were applied according to the Clinical and Laboratory Standards Institute guidelines. Turbidity of the inoculums was standardized using the 0.5 MacFarland standard. The isolates were tested for susceptibility to different antibiotics including; Aminoglycosides such as Tobramycin (10 mcg), Quinolones such as Ciprofloxacin (5mcg), Monobactams such as Aztreonam (30 mcg) and Colistin sulphate $(10 \mathrm{mcg})$, Cephems such as Ceftriaxone $(30 \mathrm{mcg})$, Cefepime $(30 \mathrm{mcg})$, Cefoxitin $(30 \mathrm{mcg})$ and Ceftazidime $(30 \mathrm{mcg})$, and Carbapenems such as Ertapenem $(10 \mathrm{mcg})$, Meropenem $(10 \mathrm{mcg})$ and Imipenem $(10 \mathrm{mcg})$. Triplicates were made for each isolate. Antibiotic discs were then placed on the Meuller hinton agar medium with a maximum of 3 discs on a plate to prevent overcrowding. Petri dishes were incubated at $37^{\circ} \mathrm{C}$ and the diameter of inhibition zone was measured between 18-24 hours of incubation. The results were interpreted according to the Clinical and Laboratory Standard Institute [10] disk diffusion standard.

\section{Results and discussion}

\section{Distribution of $K$. pneumoniae with respect to site of isolation}

The results of the distribution of clinical isolates of $K$. pneumoniae from the different isolation sites were as follows: $37 \%$ from leg, $18 \%$ from cesarean section, $20 \%$ from arm, $10 \%$ from head, $3 \%$ from buttock, $4 \%$ from breast, and $8 \%$ from thigh, as shown in Figure-1 below. 


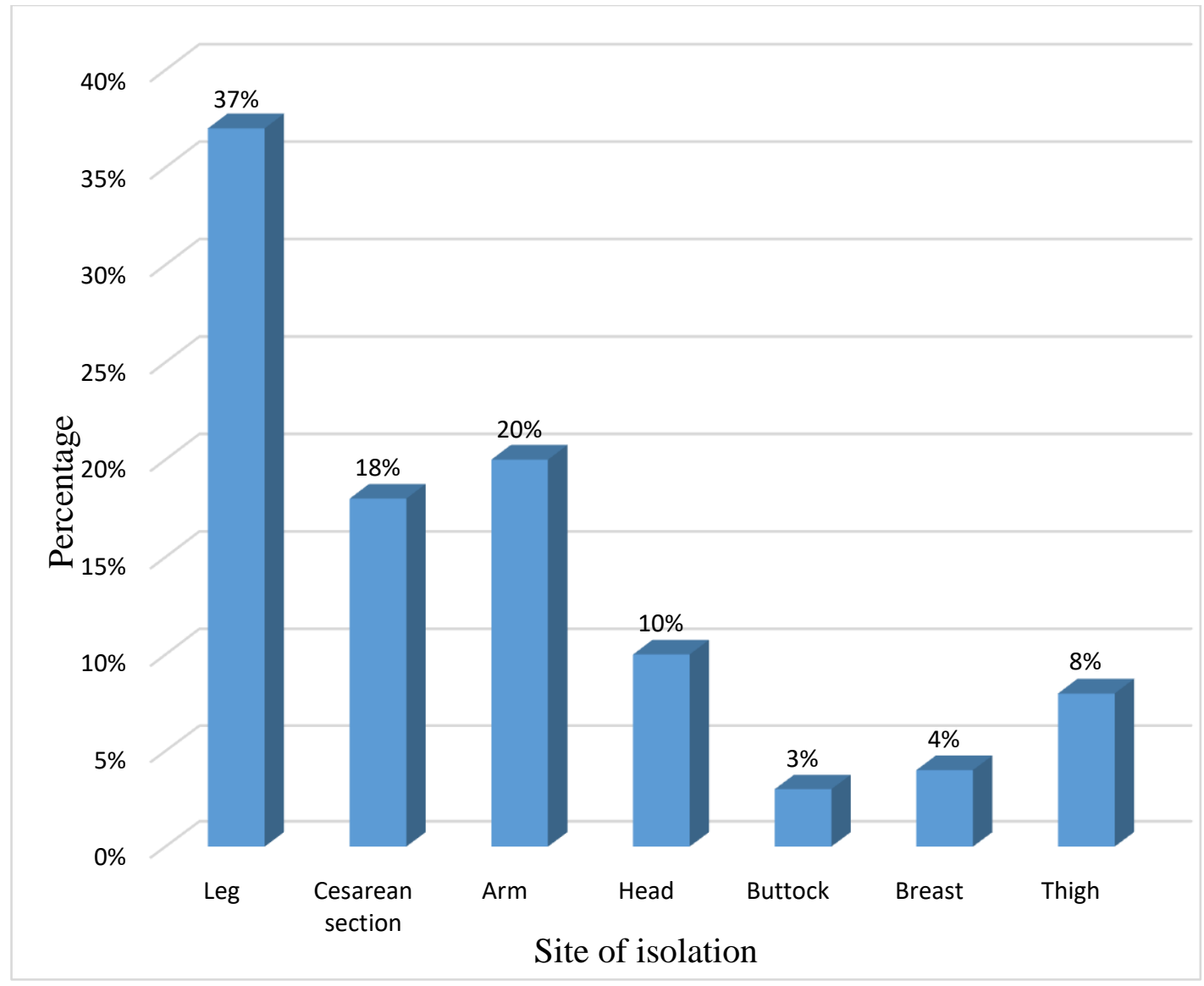

Figure 1-Distribution of $K$. pneumoniae with respect to site of isolation.

\section{Antimicrobial susceptibility testing results}

Antimicrobial susceptibility testing revealed a susceptibility rate ranging between $5 \%$ and $99 \%$, while the resistance ranged between $2 \%$ and $97 \%$.

For the Monobactams class of antibiotics, $25 \%$ of the isolates were susceptible to Aztreonam, while $40 \%$ were resistant. There was also a $35 \%$ pandrug resistance. Considering Cephems, the overall susceptibility ranged between $27 \%$ and $55 \%$ while the resistance ranged between $15 \%$ and $91 \%$. The average sensitivity was recorded to be higher using Ceftazidime (55\%), followed by Cefoxitin (53\%). The highest resistance was recorded to Ceftriaxime (91\%). The highest pandrug resistance was recorded to Cefepime (49\%) (Table-1).

For Tobramycin, $73 \%$ of the isolates were resistant and $17 \%$ were sensitive, while none of the isolates were pandrug resistant. K. pneumoniae showed high resistance to Ciprofloxacin (97\%) while only $3 \%$ were sensitive. The antibiogram activity of Colistin Sulfate could not be interpreted according to the Clinical and Laboratory Standard Institute [10] disk diffusion standard (Table- 2).

Among Carbapenems, all isolates were sensitive to Imipenem (99\%), followed by Meropenem (96\%) and Ertapenem (91\%). There was 3\% pandrug resistance recorded for Ertapenem. The least resistance was recorded to Meropenem (2\%) (Table-2).

The usual antibiotic treatments for $K$. pneumoniae infections include $\beta$-lactams such as Cephalosporins, Aminoglycosides such as Gentamycin, and Quinolones [2]. However, these treatments were found to be ineffective against certain strains that contain effective resistance mechanisms $[3,11]$. Hence, this study was carried out to evaluate the effectiveness of Carbapenems to clinical isolates of $K$. pneumoniae.

For Aztreonam, the overall resistance was $40 \%$. This trend corroborates with the results of an earlier report [12]. For Cephems, poor susceptibility was recorded for Ceftriaxime (5\%) and Cefepime (29\%). Average susceptibility was recorded for Ceftazidime (55\%) and Cefoxitin (53\%). This disagrees with data from previous reports $[13,14]$ with higher susceptibility rates of $94 \%$ and $97.7 \%$, respectively, for Cefepime. The present results also disagree with other findings [15] that reported 84.5\% 
susceptibility for Ceftazidime. The low susceptibility to Ceftriaxime and Cefepime along with the average susceptibility to Ceftazidime and Cefoxitin recorded in the recent study could be due to the fact that these antibiotics are generally available and affordable. Hence, they are frequently used by patients even without the physician's prescription, resulting into misappropriate use of these drugs. This therefore tends to increase the resistance rate to these antibiotics, thereby reducing the susceptibility rate.

Most of the isolates were found to be highly resistant to Ciprofloxacin (97\%), Ceftriaxone (91\%) and Tobramycin (73\%), which is in accordance with previously published findings [16]. Result obtained in this study showed higher percentage of isolate's resistance to Ciprofloxacin compared to the findings of Chakraborty et al., [17] where 37.5\% resistance to Ciprofloxacin was recorded. None of the isolates showed any pandrug resistance to Aminoglycosides and Quinolones. The antibiogram of Colistin Sulfate could not be interpreted according to the Clinical and Laboratory Standard Institute [10] guidelines.

The antibiogram showed that all the isolates of $K$. pneumoniae were highly sensitive to all the Carbapenems; Imipenem (99 \%), Meropenem (96 \%) and Ertapenem (91\%). This corroborates the results from earlier reports [18-21] where Meropenem and Imipenem were found to be the most effective antibiotics, with susceptibility rates ranging from $69 \%$ to $100 \%$.

The low resistance rate to Carbapenems observed in the present study could be due to their low level of usage in Nigeria because of their expensiveness. Also, Carbapenems have been found to be stable in the presence of wide range of $\beta$-lactamases [22]. Hence, the result shows that this class of drugs remains the drug of choice in treating severe $K$. pneumoniae infections.

Also, $2 \%$ of the isolates showed extreme drug resistance to Ertapenem, which is in accordance with previously reported data [23]

Table 1- Antibiotic susceptibility pattern of K. pneumoniae isolated from wounds of different clinical sites at the Department of Medical Microbiology and Parasitology of The University College Hospital (UCH), Ibadan, Oyo State, Nigeria to Monobactams and Cephems.

\begin{tabular}{|c|c|c|c|c|c|c|c|c|c|c|c|c|c|c|c|}
\hline \multirow{3}{*}{$\begin{array}{c}\text { SITE } \\
\text { OF } \\
\text { ISOL } \\
\text { ATIO } \\
\text { N (N) }\end{array}$} & \multirow{2}{*}{\multicolumn{3}{|c|}{$\begin{array}{c}\text { MONOBACTAMS } \\
\text { Aztreonam }\end{array}$}} & \multicolumn{12}{|c|}{ CEPHEMS } \\
\hline & & & & \multicolumn{3}{|c|}{ Cefepime } & \multicolumn{3}{|c|}{ Ceftriaxone } & \multicolumn{3}{|c|}{ Cefoxitin } & \multicolumn{3}{|c|}{ Ceftazidime } \\
\hline & $\mathbf{S}(\%)$ & $\begin{array}{c}\text { I } \\
(\%)\end{array}$ & $\begin{array}{c}\mathbf{R} \\
(\%)\end{array}$ & $\begin{array}{c}S \\
(\%)\end{array}$ & $\begin{array}{c}I \\
(\%)\end{array}$ & $\begin{array}{c}\mathbf{R} \\
(\%)\end{array}$ & $\begin{array}{c}\mathbf{S} \\
(\%)\end{array}$ & $\begin{array}{c}I \\
(\%)\end{array}$ & $\begin{array}{c}\mathrm{R} \\
(\%)\end{array}$ & $\begin{array}{c}\mathbf{S} \\
(\%)\end{array}$ & $\begin{array}{c}I \\
(\%)\end{array}$ & $\begin{array}{c}\mathbf{R} \\
(\%)\end{array}$ & $\underset{(\%)}{S}$ & $\begin{array}{c} \\
(\%)\end{array}$ & $\begin{array}{c}\mathbf{R} \\
(\%)\end{array}$ \\
\hline $\begin{array}{c}\text { LEG } \\
(37)\end{array}$ & $\begin{array}{c}9 \\
(24.3 \% \\
)\end{array}$ & $\begin{array}{c}12 \\
(32.4 \\
)\end{array}$ & $\begin{array}{c}16 \\
(44.4 \\
)\end{array}$ & $\begin{array}{c}6 \\
(16 . \\
6)\end{array}$ & $\begin{array}{c}19 \\
(52.7 \\
)\end{array}$ & $\begin{array}{c}12 \\
(32.4 \\
)\end{array}$ & $\begin{array}{c}3 \\
(8.1)\end{array}$ & - & $\begin{array}{c}34 \\
(91.9)\end{array}$ & $\begin{array}{c}22 \\
(59.5 \\
)\end{array}$ & $\begin{array}{c}3 \\
(8.1)\end{array}$ & $\begin{array}{c}12 \\
(32.4 \\
)\end{array}$ & $\begin{array}{c}21 \\
(56.8 \\
)\end{array}$ & $\begin{array}{l}10 \\
(27 . \\
0)\end{array}$ & $\begin{array}{c}6 \\
(16.6)\end{array}$ \\
\hline $\begin{array}{c}\text { CESA } \\
\text { REAN } \\
\text { SECTI } \\
\text { ON } \\
(18) \\
\end{array}$ & $\begin{array}{c}5 \\
(27.7)\end{array}$ & $\begin{array}{c}6 \\
(33.3 \\
)\end{array}$ & $\begin{array}{c}7 \\
(38.8 \\
)\end{array}$ & $\begin{array}{c}4 \\
(22 . \\
2)\end{array}$ & $\begin{array}{c}9 \\
(50.0 \\
)\end{array}$ & $\begin{array}{c}5 \\
(27.7 \\
)\end{array}$ & - & $\begin{array}{c}1 \\
(5.5)\end{array}$ & $\begin{array}{c}17 \\
(94.4)\end{array}$ & $\begin{array}{c}8 \\
(44.4 \\
)\end{array}$ & $\begin{array}{c}4 \\
(22 . \\
2)\end{array}$ & $\begin{array}{c}6 \\
(33.3 \\
)\end{array}$ & $\begin{array}{c}6 \\
(33.3 \\
)\end{array}$ & $\begin{array}{c}9 \\
(50 . \\
0)\end{array}$ & $\begin{array}{c}3 \\
(16.6)\end{array}$ \\
\hline$\underset{(\mathbf{2 0})}{\mathbf{A R M}}$ & $\begin{array}{c}7 \\
(35.0)\end{array}$ & $\begin{array}{c}5 \\
(25.0 \\
\quad) \\
\end{array}$ & $\begin{array}{c}8 \\
(40.0 \\
\quad) \\
\end{array}$ & $\begin{array}{c}6 \\
(30 . \\
0) \\
\end{array}$ & $\begin{array}{c}12 \\
(60.0 \\
) \\
\end{array}$ & $\begin{array}{c}2 \\
(10.0 \\
)\end{array}$ & - & $\begin{array}{c}2 \\
(10 . \\
0)\end{array}$ & $\begin{array}{c}18 \\
(90.0)\end{array}$ & $\begin{array}{c}10 \\
(50.0 \\
)\end{array}$ & $\begin{array}{c}5 \\
(25 . \\
0)\end{array}$ & $\begin{array}{c}5 \\
(25.0 \\
) \\
\end{array}$ & $\begin{array}{c}14 \\
(70.0 \\
)\end{array}$ & $\begin{array}{c}4 \\
(20 . \\
0) \\
\end{array}$ & $\begin{array}{c}2 \\
(10.0)\end{array}$ \\
\hline $\begin{array}{c}\text { HEAD } \\
\text { (10) }\end{array}$ & $\begin{array}{c}3 \\
(30.0)\end{array}$ & $\begin{array}{c}3 \\
(30.0 \\
\quad) \\
\end{array}$ & $\begin{array}{c}4 \\
(40.0 \\
\quad) \\
\end{array}$ & $\begin{array}{c}5 \\
(50 . \\
0)\end{array}$ & $\begin{array}{c}1 \\
(10.0 \\
)\end{array}$ & $\begin{array}{c}4 \\
(40.0 \\
\quad) \\
\end{array}$ & - & $\begin{array}{c}1 \\
(10 . \\
0)\end{array}$ & $\begin{array}{c}9 \\
(90.0)\end{array}$ & $\begin{array}{c}4 \\
(40.0 \\
\quad) \\
\end{array}$ & $\begin{array}{c}3 \\
(30 . \\
0)\end{array}$ & $\begin{array}{c}3 \\
(30.0 \\
\quad)\end{array}$ & $\begin{array}{c}3 \\
(30.0 \\
)\end{array}$ & $\begin{array}{c}5 \\
(50 . \\
0)\end{array}$ & $\begin{array}{c}1 \\
(10.0)\end{array}$ \\
\hline $\begin{array}{c}\text { BUTT } \\
\text { OCK } \\
(3)\end{array}$ & - & $\begin{array}{c}2 \\
(66.6 \\
)\end{array}$ & $\begin{array}{c}1 \\
(33.3 \\
)\end{array}$ & $\begin{array}{c}2 \\
(66 . \\
6) \\
\end{array}$ & 1 & - & - & - & $\begin{array}{c}3 \\
(100)\end{array}$ & $\begin{array}{c}3 \\
(100 . \\
0)\end{array}$ & - & - & $\begin{array}{c}3 \\
(100 . \\
0)\end{array}$ & - & - \\
\hline $\begin{array}{l}\text { BREA } \\
\text { ST (4) }\end{array}$ & - & $\begin{array}{c}2 \\
(50.0 \\
)\end{array}$ & $\begin{array}{c}2 \\
(50.0 \\
)\end{array}$ & $\begin{array}{c}2 \\
(50 . \\
0)\end{array}$ & $\begin{array}{c}2 \\
(50.0 \\
)\end{array}$ & - & $\begin{array}{c}1 \\
(25 . \\
0)\end{array}$ & - & $\begin{array}{c}3 \\
(75.0)\end{array}$ & $\begin{array}{c}1 \\
(25.0 \\
)\end{array}$ & $\begin{array}{c}2 \\
(50 . \\
0)\end{array}$ & $\begin{array}{c}1 \\
(25.0 \\
)\end{array}$ & $\begin{array}{c}4 \\
(100 . \\
0)\end{array}$ & - & - \\
\hline $\begin{array}{l}\text { THIG } \\
\text { H (8) }\end{array}$ & $\begin{array}{c}1 \\
(12.5)\end{array}$ & $\begin{array}{c}5 \\
(62.5 \\
\quad \\
\end{array}$ & $\begin{array}{c}2 \\
(25.0 \\
\quad\end{array}$ & $\begin{array}{c}2 \\
(25 . \\
0)\end{array}$ & $\begin{array}{c}5 \\
(62.5 \\
\quad \\
\end{array}$ & $\begin{array}{c}1 \\
(12.5 \\
)\end{array}$ & $\begin{array}{c}1 \\
(12 . \\
5)\end{array}$ & - & $\begin{array}{c}7 \\
(87.5)\end{array}$ & $\begin{array}{c}5 \\
(62.5 \\
\quad) \\
\end{array}$ & - & $\begin{array}{c}3 \\
(37.5 \\
) \\
\end{array}$ & $\begin{array}{c}4 \\
(50.0 \\
\quad \\
\end{array}$ & $\begin{array}{c}2 \\
(25 . \\
0)\end{array}$ & $\begin{array}{c}2 \\
(25.0)\end{array}$ \\
\hline $\begin{array}{c}\text { SUSC } \\
\text { EPTIB } \\
\text { ILITY } \\
(\%)\end{array}$ & 25 & 35 & 40 & 27 & 49 & 24 & 5 & 4 & 91 & 53 & 17 & 30 & 55 & 30 & 14 \\
\hline
\end{tabular}

Key: ATM Aztreonam; FEP Cefepime; CRO Ceftriaxone; FOX Cefoxitin; CAZ Ceftaxidime; S Sensitive; I Intermediate; R Resistant. 
Table 2-Antibiotic susceptibility pattern of clinical isolates of $K$. pneumoniae to Aminoglycosides, Quinolones and Carbapenems

\begin{tabular}{|c|c|c|c|c|c|c|c|c|c|c|c|c|c|c|c|c|c|c|}
\hline \multirow{3}{*}{$\begin{array}{l}\text { SITE } \\
\text { OF } \\
\text { INFE } \\
\text { CTIO } \\
\text { N (N) }\end{array}$} & \multirow{2}{*}{\multicolumn{3}{|c|}{$\begin{array}{c}\text { AMINOGLYCOSID } \\
\text { ES } \\
\text { TOB }\end{array}$}} & \multicolumn{6}{|c|}{ QUINOLONES } & \multicolumn{9}{|c|}{ CARBAPENEMS } \\
\hline & & & & \multicolumn{3}{|c|}{ CIP } & \multicolumn{3}{|c|}{ CT } & \multicolumn{3}{|c|}{ ETP } & \multicolumn{3}{|c|}{ MEM } & \multicolumn{3}{|c|}{ IPM } \\
\hline & $\begin{array}{c}\mathbf{S} \\
(\%)\end{array}$ & I $(\%)$ & $\begin{array}{c}R \\
(\%)\end{array}$ & $\begin{array}{l}\mathbf{S} \\
( \\
\% \\
\end{array}$ & $\begin{array}{l}\text { I } \\
( \\
\% \\
\end{array}$ & $\begin{array}{c}\mathbf{R} \\
(\%)\end{array}$ & $\begin{array}{c}S \\
(\% \\
)\end{array}$ & $\begin{array}{c}\text { I } \\
(\% \\
)\end{array}$ & $\begin{array}{c}\mathbf{R} \\
(\% \\
)\end{array}$ & $\underset{(\%)}{S}$ & $\begin{array}{c}I \\
(\%)\end{array}$ & $\begin{array}{c}\mathbf{R} \\
(\% \\
)\end{array}$ & $\underset{(\%)}{\mathbf{S}}$ & $\begin{array}{l}\text { I } \\
( \\
\% \\
\end{array}$ & $\begin{array}{c}\mathbf{R} \\
(\% \\
)\end{array}$ & $\begin{array}{c}\mathbf{S} \\
(\%)\end{array}$ & $\begin{array}{l}\text { I } \\
( \\
\% \\
\end{array}$ & $\begin{array}{c}\mathbf{R} \\
( \\
\% \\
) \\
\end{array}$ \\
\hline $\begin{array}{c}\text { LEG } \\
(37)\end{array}$ & $\begin{array}{c}4 \\
(10.8 \\
\quad \\
\end{array}$ & - & $\begin{array}{c}31 \\
(83.8 \\
\quad) \\
\end{array}$ & - & - & $\begin{array}{c}37 \\
(100 . \\
0)\end{array}$ & ND & ND & ND & $\begin{array}{c}33 \\
(89.2)\end{array}$ & $\begin{array}{c}1 \\
(2.7 \\
)) \\
\end{array}$ & $\begin{array}{c}3 \\
(8.1 \\
) \\
\end{array}$ & $\begin{array}{c}36 \\
(97.3 \\
\quad \\
\end{array}$ & & $\begin{array}{l}1 \\
(2 . \\
7) \\
\end{array}$ & $\begin{array}{c}36 \\
(97.3 \\
\quad \\
\end{array}$ & - & - \\
\hline $\begin{array}{l}\text { CESA } \\
\text { REAN } \\
\text { SECTI } \\
\text { ON } \\
(18)\end{array}$ & $\begin{array}{c}2 \\
(11.1 \\
)\end{array}$ & - & $\begin{array}{c}12 \\
(66.6 \\
)\end{array}$ & $\begin{array}{l}1 \\
(5 . \\
5)\end{array}$ & - & $\begin{array}{c}17 \\
(94.4 \\
)\end{array}$ & ND & ND & ND & $\begin{array}{c}16 \\
(88.8)\end{array}$ & $\begin{array}{c}2 \\
(11 . \\
1)\end{array}$ & - & $\begin{array}{c}17 \\
(94.4 \\
)\end{array}$ & & - & $\begin{array}{c}18 \\
(100 . \\
0)\end{array}$ & - & - \\
\hline $\begin{array}{c}\text { ARM } \\
\text { (20) }\end{array}$ & $\begin{array}{c}6 \\
(30.0 \\
\quad) \\
\end{array}$ & - & $\begin{array}{c}13 \\
(65.0 \\
\quad) \\
\end{array}$ & $\begin{array}{r}1 \\
(5 . \\
0) \\
\end{array}$ & - & $\begin{array}{c}19 \\
(95.0 \\
\quad \\
\end{array}$ & ND & ND & ND & $\begin{array}{c}18 \\
(90.0) \\
\end{array}$ & - & $\begin{array}{c}2 \\
(10 . \\
0)\end{array}$ & $\begin{array}{c}19 \\
(95.0 \\
\quad) \\
\end{array}$ & & - & $\begin{array}{c}20 \\
(100 . \\
0) \\
\end{array}$ & - & - \\
\hline $\begin{array}{c}\text { HEAD } \\
(10)\end{array}$ & $\begin{array}{c}4 \\
(40.0 \\
\quad \\
\end{array}$ & - & $\begin{array}{c}6 \\
(66.6 \\
\quad) \\
\end{array}$ & - & - & $\begin{array}{c}9 \\
(90.0 \\
) \\
\end{array}$ & ND & ND & ND & $\begin{array}{c}10 \\
(100 . \\
0) \\
\end{array}$ & - & - & $\begin{array}{c}10 \\
(100 . \\
0)\end{array}$ & & - & $\begin{array}{c}10 \\
(100 . \\
0)\end{array}$ & - & - \\
\hline $\begin{array}{c}\text { BUTT } \\
\text { OCK } \\
\text { (3) }\end{array}$ & - & - & $\begin{array}{c}3 \\
(100 . \\
0) \\
\end{array}$ & - & - & $\begin{array}{c}3 \\
(100 . \\
0) \\
\end{array}$ & ND & ND & ND & $\begin{array}{c}3 \\
(100 . \\
0) \\
\end{array}$ & - & - & $\begin{array}{c}3 \\
(100 . \\
0) \\
\end{array}$ & & - & $\begin{array}{c}3 \\
(100 . \\
0) \\
\end{array}$ & - & - \\
\hline $\begin{array}{l}\text { BREA } \\
\text { ST (4) }\end{array}$ & $\begin{array}{c}1 \\
(25.0 \\
) \\
\end{array}$ & - & $\begin{array}{c}2 \\
(50.0 \\
\quad) \\
\end{array}$ & - & - & $\begin{array}{c}4 \\
(100 . \\
0) \\
\end{array}$ & ND & ND & ND & $\begin{array}{c}4 \\
(100 . \\
0) \\
\end{array}$ & - & - & $\begin{array}{c}3 \\
(75.0 \\
\quad) \\
\end{array}$ & - & $\begin{array}{c}1 \\
(25 \\
.0) \\
\end{array}$ & $\begin{array}{c}4 \\
(100 . \\
0) \\
\end{array}$ & - & - \\
\hline $\begin{array}{l}\text { THIG } \\
\text { H (8) }\end{array}$ & - & - & $\begin{array}{c}6 \\
(75.0 \\
\quad) \\
\end{array}$ & - & - & $\begin{array}{c}8 \\
(100 . \\
0) \\
\end{array}$ & ND & ND & ND & $\begin{array}{c}7 \\
(87.5)\end{array}$ & - & $\begin{array}{c}1 \\
(12 . \\
5)\end{array}$ & $\begin{array}{c}8 \\
(100 . \\
0)\end{array}$ & & - & $\begin{array}{c}8 \\
(100 . \\
0) \\
\end{array}$ & - & - \\
\hline $\begin{array}{c}\text { SUSC } \\
\text { EPTIB } \\
\text { ILITY } \\
(\%) \\
\end{array}$ & 17 & - & 73 & 2 & - & 97 & ND & ND & ND & 91 & 3 & 6 & 96 & - & 2 & 99 & - & - \\
\hline
\end{tabular}

TOB: Tobramycin, CIP: Ciprofloxacin, CT: Colistin sulphate, ETP: Ertapenem, MEM: Meropenem, IPM: Imipenem, S: Sensitive, I: Intermediate, R: Resistant, ND: Not determinable.

\section{Conclusions}

High levels of susceptibility recorded for Imipenem, Meropenem and Ertapenem in this study are indications that these antibiotics can still be utilized as antibiotics for infections caused by multidrug resistant pathogens. Continuous surveillance is however crucial to monitor the antimicrobial resistance among K. pneumoniae isolates.

\section{References}

1. Enwuru, N.V., Enwuru, C.A., Ogbonnia, S.O. and Adepoju-Bello, A.A. 2011. Metallo- $\beta$ lactamase production by Escherichia coli and Klebsiella species isolated from hospital and community subjects in Lagos, Nigeria. Nature Science, 9(11): 83-88.

2. Qureshi, S. 2015. Klebsiella Infections Treatment and Management (M. Bronze, Ed.). Retrieved November 29, 2015, from http://emedicine.medscape.com/article/219907-treatment. .

3. Kumar, V., Sun, P., Vamathevan, J., Li, Y., Ingraham, K., Palmer, L. and Brown, J.R. 2011. Comparative Genomics of Klebsiella pneumoniae Strains with Different Antibiotic Resistance Profiles. Journal of Antimicrobial Agents and Chemotherapy, 2(2):15.

4. Vuotto, C., Longo, F., Balice, M. P., Donelli, G. and Varaldo, P. E. 2014. Antibiotic Resistance Related to Biofilm Formation in Klebsiella pneumoniae. Pathogens. 3(3): 743-758.

5. Peleg, A.Y. and Hooper, D.C. 2010. Hospital-acquired infections due to gram-negative bacteria: a review. The New England Journal of Medicine, 362: 1804-13.

6. Papp-Wallace, K. M., Endimiani, A., Taracila, M. A. and Bonomo, R. A. 2011. Carbapenems: Past, Present, and Future . Journal of Antimicrobial Agents and Chemotherapy, 55(11): 49434960. 
7. Brisse, S., Fevre, C., Passet, V., Issenhuth-Jeanjean, S., Tournebize, R., Diancourt, L. and Grimont, P. 2009. Virulent clones of Klebsiella pneumoniae: identification and evolutionary scenario based on genomic and phenotypic characterization. PLoS One, 4, e4982.

8. Morrill, J.J., Pogue, J.M., Kaye, K.S., and LaPlante, K.L. 2015. Treatment Options for Carbapenem-resistant Enterobacteriaceae Infections. Open Forum Infect Dis.; 2(2): 50.

9. Cheesbrough, M. 2002. District laboratory practice in tropical countries. Part II; Cambridge University Press UK. pp. 136-142.

10. Clinical and Laboratory Standards Institute. 2017. Performance Standards for Antimicrobial Susceptibility Testing. Wayne, PA: CLSI; Document M100.

11. Bassetti, M., Russo, A., Carnelutti, A. and La Rosa, A., Righi E. 2018. Antimicrobial resistance and treatment: an unmet clinical safety need. Journal of Expert Opinion on Drug Safety, 17(7): 669-680.

12. Namratha, K.G 2015. Characterization and antibiogram of Klebsiella spp. Isolated from clinical specimen in a rural teaching hospital. Scholars Journal of Applied Medical Sciences, 3(2): 878883.

13. Bouchillon, S.K., Badal, R.E., Hoban, D.J. and Hawser, S.P. 2013. Antimicrobial susceptibility of inpatient urinary tract isolates of gram-negative bacilli in the United States: results from the study for monitoring antimicrobial resistance trends (SMART) program: 2009-2011. Clinical therapeutics, 35: 872-877.

14. Ishikawa, K. 2015. Japanese nationwide surveillance in 2011 of antibacterial susceptibility patterns of clinical isolates from complicated urinary tract infection cases. Journal of infection and chemotherapy: official journal of the Japan Society of Chemotherapy, 21: 623-633.

15. Liu, W.P., Wang, J.T., Chang, S.C., Chang, F.Y., Fung, C.P., Chaung, Y.C., Shiau, Y.R., Tan, M.C., Wang, H.Y., Lai, J.F., Huang, I.W. and Lauderdale, T.L. 2016. The antimicrobial susceptibility of Klebsiella pneumoniae from community settings in Taiwan, a trend analysis. Scientific Reports, 6: 36-40.

16. Bina, M., Pournajaf, A., Mirkalantari, S., Talebi, M. and Irajian, G. 2015. Detection of the Klebsiella pneumoniae carbapenemase (KPC) in K. pneumoniae isolated from the clinical samples by the phenotypic and genotypic methods. Iran Journal of Pathology, 10(3): 199-205.

17. Chakraborty, S., Mohsina, K., Sarker, P.K., Alam, M.Z., Abdul Karim, M.I., and Abu Sayem, S.M. 2016. Prevalence, antibiotic susceptibility profiles and ESBL production in Klebsiella pneumoniae and Klebsiella oxytoca among hospitalized patients. Periodicum Biologorum, 118(1): 53-58. DOI: 10.18054/pb.2016.118.1.3160.

18. Osundiya, O.O., Oladele, R.O., and Oduyebo, O.O. 2013. Multiple Antibiotic Resistance (MAR) Indices of Pseudomonas and Klebsiella species isolates in Lagos University Teaching Hospital. African Journal of Clinical and Experimental Microbiology, 14(3): 164-168.

19. Babakhani, S., Shokri, S., and Baharvand, M. 2015. Antibiotic resistance pattern of Klebsiella pnuemoniae isolated from nosocomial infections in Aleshtar hospital, Lorestan province. Report of Health Care. 1(2): 55-59.

20. Yusuf, I., Yushu'a, M., Sherif, A.A., Getso, M.I., Yahaya, H., and Bala, J.A. 2012. Detection of metallo beta lactamases among gram negative bacteria isolates from Murtala Muhammed Specialist Hospital, Kano and Al Madina Hospital Kaduna, Nigeria. Bayero Journal of Pure and Applied Science, 5(2): 84-88.

21. Hussaini, I.M., Olonitola, O.S. and Suleiman, A.B. 2017. Occurrence of Carbapenem resistant Klebsiella pneumoniae in clinical samples from some selected hospitals in Zaria, Kaduna State. Journal of Advances in Medical and Pharmaceutical Sciences, 13(3): 1-11.

22. Kattan, J.N., Villegas, M.V. and Quinn, J.P. 2008. New developments in Carbapenems. Journal of Clinical Microbiology and Infection. 14(2): 112-115.

23. Ansari, M., Munir, T. and Saad, N. 2018. Phenotypic identification, frequency distribution and antibiogram of carbapenemase producing Enterobacteriaceae in clinical isolates. Journal of the College of Physicians and Surgeons Pakistan, 28(4): 274-278. 